\title{
ANÁLISE EXPERIMENTAL MULTICRITÉRIO DE AUXÍLIO À TOMADA DE DECISÃO NA SELEÇÃO DE PESSOAL PARA UM PROJETO DE DESENVOLVIMENTO DE SOFTWARE
}

\author{
Glauco Barbosa da Silva \\ Centro de Análise de Sistemas Navais (CASNAV - UFF) \\ Praça Barão de Ladário s/n - Ilha das Cobras \\ Rio de Janeiro - RJ - CEP: 20091-000 \\ glaucos@id.uff.br \\ Phelipe Medeiros da Rocha \\ Universidade Federal Fluminense (UFF) \\ Rua Passos da Pátria, 156 - Bloco D - sala309 \\ São Domingos, Niterói, RJ - Brasil \\ phelipe m rocha@yahoo.com.br
}

\begin{abstract}
Resumo
O presente trabalho tem por objetivo propor soluções para o problema de seleção múltipla por meio de uma análise experimental multicritério baseada na Technique for Order Preference by Similarity to Ideal Solution (TOPSIS). Para isso, desenvolve-se um problema prático de seleção de uma equipe em um projeto de desenvolvimento de software utilizando TOPSIS e, em seguida, os resultados alcançados são apresentados.
\end{abstract}

Palavras-Chaves: $\quad$ TOPSIS; Apoio à Decisão Multicritério; Seleção de Pessoal.

\begin{abstract}
This paper aims to propose solutions to the problem of multiple selection through a multicriteria experimental analysis based on Technique for Order Preference by Similarity to Ideal Solution (TOPSIS). To this end, it develops a practical problem of selecting a team on a Software project using TOPSIS and then, the results obtained are presented.
\end{abstract}

Keywords:

TOPSIS; MCDA; Software Project Team Selection. 


\section{INTRODUÇÃO}

As empresas vêm sofrendo um fenômeno comum: a crescente complexidade dos projetos aliada à redução dos ciclos de vida de produtos e serviços. Tal mudança origina-se da consciência do valor estratégico da competição baseada no tempo e da necessidade de melhoria contínua da qualidade [1]. Desta maneira, a gestão de projetos tornou-se, uma questão estratégica, essencial para permanência e competição de qualquer organização no mercado.

No contexto do gerenciamento de projetos, a formação de equipes constitui um processo decisório que envolve características difíceis de serem mensuradas, envolvendo muitos aspectos subjetivos que tornam o processo bastante complexo. Para Alencar \& Almeida[2], a seleção de equipes é, na prática, um problema complexo de apoio a decisão multicritério. Muitos abordaram o problema para a seleção de empresas e profissionais para a participação em projetos diversos com métodos multicritérios, tais como: Al-Reshaid \& Kartam [3], Alencar \& Almeida [4], Al-Harbi [5] e Kelemenis, Ergazakis \& Askounis [6].

Face às peculiaridades de um projeto de desenvolvimento de software, o processo de seleção de pessoal para essa atividade requer atenção especial no que tange a sinergia da equipe, isto é, a complementaridade das alternativas pode ser mais interessante que a sobreposição delas. Considerando a interação entre critérios, busca-se escolher mais de uma alternativa, no mesmo problema decisório, partindo da hipótese que as $\underline{n}$ melhores alternativas em uma ordenação não são necessariamente o melhor conjunto de $\underline{n}$ alternativas, configurando-se um problema de seleção múltipla,

Os métodos multicritérios buscam em um espaço de alternativas possíveis uma solução capaz de melhor atender as restrições e critérios associados ao problema proposto. $\mathrm{Na}$ seleção de pessoal em projetos de software a Análise Multicritério apresenta-se como potencial candidata no auxílio à tomada de decisão.

O presente trabalho tem por objetivo propor soluções para o problema de seleção múltipla por meio de uma análise experimental multicritério baseada na Technique for Order Preference by Similarity to Ideal Solution (TOPSIS). Para isso, desenvolve-se um problema prático de seleção de uma equipe em um projeto de desenvolvimento de software utilizando TOPSIS e, em seguida, os resultados alcançados são apresentados.

\section{METODOLOGIA MULTICRITÉRIO DE AUXÍLIO À TOMADA DE DECISÃO}

Apoio à Decisão Multicritério é uma sub-disciplina e ramo crescente da Pesquisa Operacional, cujo tem forte interesse na concepção de ferramentas matemáticas e computacionais para apoiar a avaliação subjetiva de um número finito de alternativas em um numero finito de critérios de desempenho por um único tomador de decisão ou por um grupo[7].

Roy[8] define o Apoio à Decisão Multicritério (AMD) como uma tentativa de prover possíveis cursos para questões levantadas pelos agentes envolvidos no processo decisório por meio de um modelo capaz de comparar alternativas viáveis.

Sant'Anna, Rabelo \& Nogueira[9] apresentam as vantagens e desvantagens da adoção de um método de AMD, alertando que um julgamento criterioso para escolher a técnica mais adequada para uma aplicação específica faz-se necessário. Uma vez que os resultados podem ser influenciados pela seleção do método; diferentes métodos aplicáveis a um problema nem sempre conduzirão às mesmas conclusões.

Em geral, os métodos multicritério são categorizados em: métodos de ponderação, métodos ordinais, métodos baseados em funções de utilidade, métodos de relações de superação, métodos baseados na distância a uma alternativa ideal, entre outras. 
A tomada de decisão é o processo de seleção de um caminho dentre todas as alternativas disponíveis. Em quase todos os problemas a multiplicidade de critérios para julgar as alternativas é generalizada. Ou seja, para muitos desses problemas, o tomador de decisão deseja atingir mais de um objetivo ou meta na escolha do curso de ação, desde que satisfaçam as restrições ditadas pelo ambiente, processos e recursos.

Matematicamente, estes problemas podem ser representados pela eq.(1), a seguir:

$$
\begin{gathered}
\operatorname{Max} F_{1}(x), F_{2}(x), \ldots, F_{k}(x) \\
\text { s.a. } \\
x \in X=\left\{x \mid g_{i}(x) \leq 0 ; \quad i=0,1,2, \ldots, m\right\}
\end{gathered}
$$

onde $\mathrm{x}$ é um vetor $\mathrm{n}$-dimensional, $\mathrm{n}$ numero de variáveis, $\mathrm{m}$ restrições e $\mathrm{k}$ objetivos.

Devido às características/particularidades dos critérios, muitas vezes conflitantes, as decisões tornam-se complexas

\subsection{TeChNique for ORder Preference by Similarity to IDEAL SOlUtion (TOPSIS)}

O TOPSIS é um método de Apoio à Decisão Multicritério que propicia a ordenação de alternativas por similaridade à Solução Ideal Positiva (PIS - Positive Ideal Solution) e dissimilaridade à Solução Ideal Negativa (NIS - Negative Ideal Solution), medidas por meio de distâncias, geralmente, euclidiana. Para Hwang, Lai \& Liu [10], essa técnica permite a redução de um espaço de busca k-dimensional para um espaço bi-dimensional.

Desenvolvido por Hwang \& Yoon[11], e posteriormente discutido por vários, o método tem apresentado crescente interesse por pesquisadores de diferentes áreas, como: Gestão da Cadeia de Suprimentos e Logística[12], Design e Sistemas de Produção[13], Ambiental[14], Médica[15], entre outras.

Em uma busca simples do termo TOPSIS na base Scopus, é possível perceber a evolução dos trabalhos que utilizaram a técnica, totalizando dois mil, trezentos e quarenta e dois resultados obtidos entre os anos de 1983 e 2013.

A execução do método é composta por seis etapas:

- Construção da Matriz de Decisão (A);

- Normalização da Matriz de Decisão (An);

- Cálculo da Matriz Ponderada;

- Determinação da PIS(S+) e NIS(S-);

- Cálculo das distâncias de cada alternativa para PIS(S+) e NIS(S-); e

- Cálculo da Similaridade (Ci).

Segundo Sant'Anna, Rabelo \& Nogueira [9], o método possui como características principais:

- Permitir a inclusão de um número ilimitado de atributos;

- Gerar uma ordenação de alternativas, com escores que possibilitam um melhor entendimento das diferenças e similaridades entre alternativas;

- Utilizar como métrica o conceito de distâncias ao PIS e NIS; e

- Facilitar a utilização, podendo ser implementado em planilha eletrônica.

No cálculo da similaridade $\left(C_{i}\right)$, onde $C_{i} \in[0,1], C_{i}=1$ representa a PIS e $C_{i}=0$ a NIS, ou seja, quanto mais próximo de 1 mais próxima a alternativa está da PIS, e quanto mais próximo de 0 mais próxima de NIS.

\section{SELEÇÃO DE PESSOAL EM PROJETOS DE SOFTWARE}

Os projetos de desenvolvimento de software (PS) estão sujeitos, todo o tempo, a fatos que podem de alguma forma impactar seus objetivos, processos ou produtos. Estes fatos podem causar efeitos adversos e em muitos casos catastróficos ao projeto.

São comuns relatos de PS com taxas relativamente altas de atrasos, com custos que 
ultrapassaram as estimativas, cuja implementação não correspondeu ao que foi solicitado ou que foram abortados antes da sua conclusão. Segundo Boehm [16], entre 15 e 35\% de todos os PS incorrem em cancelamento ou erros de orçamento e/ou de agenda e/ou qualidade.

Relatos de experiência dos PS mostraram que muitos dos problemas poderiam ter sido evitados ou fortemente reduzidos se tivessem identificados e solucionados os elementos de maior risco. O fator humano se sobressai dentre os elementos de maior risco para um PS, em geral, a equipe técnica envolvida representa o maior capital investido na grande maioria dos casos.

Palharini[17] afirma que a Seleção Científica de Pessoas é o processo por meio do qual se procede a escolha, entre um determinado número de candidatos, daqueles que apresentem maior probabilidade de desempenhar satisfatoriamente as tarefas previstas; de se adaptarem às características do cargo e ao desenvolvimento da organização.

Nesse contexto, o recrutamento e seleção do pessoal envolvido podem ser determinantes para o sucesso do empreendimento e da organização. Portanto, a utilização de métodos de Apoio à Decisão Multicritério apresentam-se como relevante ferramental para o processo decisório.

Considerando a criticidade do processo de formação da equipe de um projeto e visando reduzir os riscos, é comum a composição de duplas de programadores. Caracteriza-se, desta forma, como um processo de seleção múltipla, ou seja, um processo em que mais de uma alternativa deve ser escolhida. Sendo assim, partindo da hipótese que as $\mathrm{n}$ melhores alternativas em uma ordenação não são necessariamente o melhor conjunto de $\underline{n}$ alternativas, na seção seguinte são descritos os passos da aplicação do método TOPSIS aos dados de um PS real, no qual se busca a seleção de um programador sênior e um júnior, à luz dos critérios descritos a seguir.

\section{METODOLOGIA E RESULTADOS OBTIDOS PELO MÉTODO NA SELEÇÃO DE PESSOAL}

O problema decisório a ser solucionado consiste da seleção de dois programadores com níveis de experiência diferentes. A partir dos dados referentes a seis candidatos, foi estabelecido que o processo seletivo seria composto de duas fases, sendo a primeira eliminatória e a segunda classificatória.

\subsection{DEFINIÇÃo dos CRITÉRIOS}

Os critérios utilizados nas duas fases foram previamente definidos de modo a atender as necessidades do projeto e da organização, sem conhecimento das alternativas. A descrição dos critérios é apresentada na tabela 1.

Tabela 1 - Descrição dos critérios.

\begin{tabular}{|l|l|}
\hline Critério & Descrição \\
\hline C1 - Idade & Quanto maior a idade, maior a estabilidade para o projeto. \\
\hline C2 - Estado Civil & Quanto mais estável, maior a estabilidade para o projeto. \\
\hline $\begin{array}{l}\text { C3 - Deslocamento para a } \\
\text { Sede }\end{array}$ & $\begin{array}{l}\text { Acessibilidade ao local de trabalho, a partir de um perímetro } \\
\text { definido. (eliminatório) }\end{array}$ \\
\hline C4 - Formação Acadêmica & Cursos realizados. \\
\hline C5 - Currículo & Clareza das informações. \\
\hline C6 - Pretensão Salarial & $\begin{array}{l}\text { Teto estabelecido pelas restrições financeiras do projeto } \\
\text { (eliminatório) }\end{array}$ \\
\hline E1 - Avaliação Global & Desempenho na entrevista. \\
\hline E2 - Apresentação Pessoal & $\begin{array}{l}\text { Função requer em algumas ocasiões o contato pessoal com o } \\
\text { cliente. }\end{array}$ \\
\hline E3 - Experiência Profissional & Participação em projetos correlatos. \\
\hline $\begin{array}{l}\text { E4 - Potencial de } \\
\text { Crescimento }\end{array}$ & Entusiasmo por temas relacionados ao projeto. \\
\hline
\end{tabular}




\subsection{PRIMEIRA FASE Da SELEÇão}

Nessa fase os dados foram coletados a partir dos currículos dos candidatos, que foram avaliados a luz dos critérios: $\mathrm{C} 1$ até $\mathrm{C} 6$. Em cada critério os candidatos foram avaliados com graus que variavam numa escala de zero até cinco.

\subsubsection{Construção da Matriz de Decisão (A)}

Na tabela 2 é apresentada a matriz de decisão A, onde cada elemento Xij representa o desempenho da alternativa Ai no critério $\mathrm{Cj}$.

Tabela 2 - Matriz de Decisão.

\begin{tabular}{|l|l|l|l|l|l|l|}
\hline \multicolumn{7}{|l|}{ Critérios } \\
\hline Alternativas & C1 & C2 & C3 & C4 & C5 & C6 \\
\hline A1 & 1.00 & 3.00 & 3.00 & 1.00 & 4.00 & 5.00 \\
\hline A2 & 1.00 & 1.00 & 5.00 & 1.00 & 3.00 & 5.00 \\
\hline A3 & 5.00 & 1.00 & 5.00 & 1.00 & 3.00 & 4.00 \\
\hline A4 & 3.00 & 1.00 & 0.00 & 2.00 & 5.00 & 0.00 \\
\hline A5 & 4.00 & 1.00 & 5.00 & 2.00 & 4.00 & 0.00 \\
\hline A6 & 5.00 & 1.00 & 3.00 & 1.00 & 4.00 & 5.00 \\
\hline
\end{tabular}

A alternativa A4 recebeu nota zero no critério $\mathrm{C} 3$ por se encontrar fora do perímetro estabelecido como aceitável em termos de distância. As alternativas A4 e A5 receberam avaliação zero no critério C6 por se encontrarem fora do limite salarial estabelecido para o cargo.

\subsubsection{Normalização da Matriz de Decisão (AN)}

Em uma abordagem linear, os valores atribuídos às alternativas em cada critério foram normalizados e encontram-se consolidados na tabela 3 .

Tabela 3 - Matriz de Decisão Normalizada.

\begin{tabular}{|l|l|l|l|l|l|l|}
\hline \multirow{2}{*}{ Alternativas } & \multicolumn{6}{l}{ Critérios } \\
\cline { 2 - 7 } & C1 & C2 & C3 & C4 & C5 & C6 \\
\hline A1 & 0.05 & 0.38 & 0.14 & 0.13 & 0.17 & 0.26 \\
\hline A2 & 0.05 & 0.13 & 0.24 & 0.13 & 0.13 & 0.26 \\
\hline A3 & 0.26 & 0.13 & 0.24 & 0.13 & 0.13 & 0.21 \\
\hline A4 & 0.16 & 0.13 & 0.00 & 0.25 & 0.22 & 0.00 \\
\hline A5 & 0.21 & 0.13 & 0.24 & 0.25 & 0.17 & 0.00 \\
\hline A6 & 0.26 & 0.13 & 0.14 & 0.13 & 0.17 & 0.26 \\
\hline
\end{tabular}

\subsubsection{Vetor de pesos dos critérios e Matriz Ponderada}

Seja w o vetor de pesos dos critérios, onde w somatório dos pesos em cada critério observa o apresentado na equação 2 . A tabela 4 apresenta os valores de $\mathrm{w}$ arbitrados.

$$
\sum_{i=1}^{n} w_{i}=1
$$


Tabela 4 - Vetor de Pesos(w).

\begin{tabular}{|l|l|l|l|l|l|l|}
\hline Vetor de pesos & C1 & C2 & C3 & C4 & C5 & C6 \\
\hline W & 0.1304 & 0.1304 & 0.21739 & 0.087 & 0.21739 & 0.21739 \\
\hline
\end{tabular}

A matriz ponderada(Ap) (tabela 5) é obtida pela multiplicação da matriz normalizada pelo vetor de pesos(w).

Tabela 5 - Matriz de Decisão Ponderada.

\begin{tabular}{|l|l|l|l|l|l|l|}
\hline \multirow{2}{*}{ Alternativas } & \multicolumn{6}{|l}{ Critérios } \\
\cline { 2 - 7 } & C1 & C2 & C3 & C4 & C5 & C6 \\
\hline A1 & 0.01 & 0.05 & 0.03 & 0.01 & 0.04 & 0.06 \\
\hline A2 & 0.01 & 0.02 & 0.05 & 0.01 & 0.03 & 0.06 \\
\hline A3 & 0.03 & 0.02 & 0.05 & 0.01 & 0.03 & 0.05 \\
\hline A4 & 0.02 & 0.02 & 0.00 & 0.02 & 0.05 & 0.00 \\
\hline A5 & 0.03 & 0.02 & 0.05 & 0.02 & 0.04 & 0.00 \\
\hline A6 & 0.03 & 0.02 & 0.03 & 0.01 & 0.04 & 0.06 \\
\hline
\end{tabular}

\subsubsection{Determinação da PIS(S+) e NIS(S-)}

A PIS(S+) e a NIS(S-) são determinadas, respectivamente, pelo maior e menor valor obtidos da matriz ponderada em cada critério. Os valores obtidos são apresentados na tabela 6.

Tabela 6 - Solução Ideal Positiva e Solução Ideal Negativa.

\begin{tabular}{|l|l|l|l|l|l|l|}
\cline { 2 - 7 } \multicolumn{1}{c|}{} & \multicolumn{5}{l|}{ Critérios } & \multicolumn{5}{l|}{} \\
\cline { 2 - 7 } \multicolumn{1}{c|}{} & C1 & C2 & C3 & C4 & C5 & C6 \\
\hline S+ & 0.03 & 0.05 & 0.05 & 0.02 & 0.05 & 0.06 \\
\hline S- & 0.01 & 0.02 & 0.00 & 0.01 & 0.03 & 0.00 \\
\hline
\end{tabular}

\subsubsection{Cálculo das distâncias de cada alternativa para PIS(S+) e NIS(S-)}

O cálculo das distâncias euclidianas de cada alternativa Ai para $\mathrm{S}+$ e $\mathrm{S}$ - é dado pelas equações (3) e (4), respectivamente. A tabela 7 consolida os valores obtidos.

$$
\begin{aligned}
& d^{+}=\sqrt{\sum_{i=1}^{n}\left(p_{i j}-p_{i}^{+}\right)^{2}} \\
& d^{-}=\sqrt{\sum_{i=1}^{n}\left(p_{i j}-p_{i}^{-}\right)^{2}}
\end{aligned}
$$

Tabela 7 - distâncias das alternativas para S+e S-.

\begin{tabular}{|l|c|c|c|c|c|c|}
\hline \multirow{2}{*}{ Distâncias } & \multicolumn{6}{|l|}{ Alternativas } \\
\cline { 2 - 7 } & A1 & A2 & A3 & A4 & A5 & A6 \\
\hline $\mathrm{d}+$ & 0.03729 & 0.04788 & 0.04086 & 0.08487 & 0.06688 & 0.04122 \\
\hline $\mathrm{d}-$ & 0.07342 & 0.07715 & 0.07435 & 0.02577 & 0.05754 & 0.07128 \\
\hline
\end{tabular}




\subsubsection{Cálculo da Similaridade} na tabela 8 .

A Similaridade (Ci) é dada pela equação (5), os resultados obtidos são apresentados

$$
C_{i}=\frac{d^{-}}{d^{+}+d^{-}}
$$

Tabela 8 - Similaridades das alternativas.

\begin{tabular}{|l|l|l|l|l|l|l|}
\hline & A1 & A2 & A3 & A4 & A5 & A6 \\
\hline Similaridade & 0.6632 & 0.6170 & 0.6453 & 0.2329 & 0.4625 & 0.6336 \\
\hline
\end{tabular}

A partir das similaridades obtidas, as alternativas foram ordenadas em: A1, A3, A6, A2, A5 e A4. Como o critério de eliminação da primeira fase era a exclusão dos dois piores desempenhos em similaridade, as alternativas A4 e A5 foram eliminadas e não participaram da fase seguinte.

\subsection{SEGUNDA FASE DA SELEÇão}

Essa fase foi dividida em duas etapas. Os dados computados foram obtidos a partir de entrevistas realizadas com os candidatos selecionados na primeira fase, que foram avaliados a luz dos critérios: E1 até E4.

Em cada critério os candidatos foram avaliados com graus que variavam numa escala de zero até cinco. Para atender ao requisito da seleção de dois profissionais com nível de experiência diferente, a primeira etapa contemplou maior peso para os critérios E1 e E3; a segunda etapa contemplou maior peso para os critérios E1 e E4.

Seguiram-se similarmente os passos da primeira fase para determinação das similaridades nas duas etapas dessa segunda fase.

\subsubsection{Primeira Etapa}

\subsubsection{Construção da Matriz de Decisão (A)}

Na tabela 9 é apresentada a matriz de decisão A, onde cada elemento Xij representa o desempenho da alternativa Ai no critério $\mathrm{Ej}$.

Tabela 9 - Matriz de Decisão.

\begin{tabular}{|l|l|l|l|l|}
\hline \multicolumn{4}{|l|}{ Critérios } & \multicolumn{3}{l|}{} \\
\hline Alternativas & E1 & E2 & E3 & E4 \\
\hline A1 & 3.50 & 3.00 & 5.00 & 4.00 \\
\hline A3 & 2.50 & 3.00 & 5.00 & 3.00 \\
\hline A6 & 2.50 & 4.00 & 5.00 & 4.00 \\
\hline A2 & 3.50 & 4.00 & 2.00 & 5.00 \\
\hline
\end{tabular}

\subsubsection{Normalização da Matriz de Decisão (AN)}

Os valores atribuídos às alternativas em cada critério(tabela 9) foram normalizados e encontram-se consolidados na tabela 10. 
Tabela 10 - Matriz de Decisão Normalizada.

\begin{tabular}{|l|l|l|l|l|}
\hline \multicolumn{4}{|l|}{ Critérios } \\
\hline Alternativas & E1 & E2 & E3 & E4 \\
\hline A1 & 0.292 & 0.214 & 0.294 & 0.250 \\
\hline A3 & 0.208 & 0.214 & 0.294 & 0.188 \\
\hline A6 & 0.208 & 0.286 & 0.294 & 0.250 \\
\hline A2 & 0.292 & 0.286 & 0.118 & 0.313 \\
\hline
\end{tabular}

\subsubsection{Vetor de pesos dos critérios e Matriz Ponderada}

O vetor de pesos $(\mathrm{w})$ com os valores dos pesos arbitrados para os critérios encontramse consolidados na tabela 11 .

Tabela 11 - Vetor de Pesos(w).

\begin{tabular}{|l|l|l|l|l|}
\hline Vetor de Pesos & E1 & E2 & E3 & E4 \\
\hline $\mathrm{W}$ & 0.455 & 0.091 & 0.273 & 0.182 \\
\hline
\end{tabular}

Multiplicando-se a matriz normalizada pelo vetor de pesos(w) obtém-se a matriz de decisão ponderada(Ap), conforme tabela 12 .

Tabela 12 - Matriz de Decisão Ponderada.

\begin{tabular}{|l|l|l|l|l|}
\cline { 2 - 5 } & \multicolumn{4}{|l|}{ Critérios } \\
\hline Alternativas & E1 & E2 & E3 & E4 \\
\hline A1 & 0.13258 & 0.01948 & 0.08021 & 0.04545 \\
\hline A3 & 0.09470 & 0.01948 & 0.08021 & 0.03409 \\
\hline A6 & 0.09470 & 0.02597 & 0.08021 & 0.04545 \\
\hline A2 & 0.13258 & 0.02597 & 0.03209 & 0.05682 \\
\hline
\end{tabular}

\subsubsection{Determinação da PIS(S+) e NIS(S-)}

A partir da tabela 11, a PIS(S+) e a NIS(S-) são determinadas, respectivamente, pelo maior e menor valor presente da matriz ponderada em cada critério. Os valores obtidos são apresentados na tabela 13 .

Tabela 13 - Solução Ideal Positiva e Solução Ideal Negativa.

\begin{tabular}{|c|c|c|c|c|}
\hline & \multicolumn{4}{|c|}{ Critérios } \\
\hline & E1 & E2 & E3 & E4 \\
\hline $\mathrm{S}+$ & 0.133 & 0.026 & 0.080 & 0.057 \\
\hline S- & 0.095 & 0.019 & 0.032 & 0.034 \\
\hline
\end{tabular}

\subsubsection{Cálculo das distâncias de cada alternativa para PIS(S+) e NIS(S-)}

O cálculo das distâncias euclidianas de cada alternativa Ai para S+e S- é dado pelas equações (3) e (4), respectivamente. A tabela 14 consolida os valores obtidos.

Tabela 14 - distâncias das alternativas para S+e S-.

\begin{tabular}{|l|c|c|c|c|}
\hline \multirow{2}{*}{ Distâncias } & \multicolumn{4}{|l|}{ Alternativas } \\
\cline { 2 - 5 } & A1 & A3 & A6 & A2 \\
\hline $\mathrm{d}+$ & 0.0130881 & 0.0446486 & 0.0395466 & 0.0481283 \\
\hline $\mathrm{d}-$ & 0.0622918 & 0.0481283 & 0.0498762 & 0.0446486 \\
\hline
\end{tabular}




\subsubsection{Cálculo da Similaridade}

na tabela 15 .

A Similaridade (Ci) é dada pela equação (5), os resultados obtidos são apresentados Tabela 15 - Similaridades das alternativas.

\begin{tabular}{|l|l|l|l|l|}
\hline & A1 & A3 & A6 & A2 \\
\hline Similaridade & 0.8263718 & 0.5187533 & 0.557757 & 0.4812467 \\
\hline
\end{tabular}

A partir das similaridades obtidas, as alternativas foram ordenadas em: A1, A3, A6, A2; sendo assim a alternativa A1, com maior valor de similaridade, foi selecionada para a composição da equipe e não participou da etapa seguinte.

\subsubsection{Segunda Etapa}

Após a retirada da alternativa $\mathrm{A} 1$, as etapas do TOPSIS são repetidas de modo a obter-se uma nova ordenação para as alternativas a partir de um novo vetor de pesos(w) arbitrado.

\subsubsection{Construção da Matriz de Decisão (A)}

Na tabela 16 é apresentada a matriz de decisão para a segunda etapa.

Tabela 16 - Matriz de Decisão.

\begin{tabular}{|l|l|l|l|l|}
\hline \multicolumn{5}{|l|}{ Critérios } \\
\hline Alternativas & E1 & E2 & E3 & E4 \\
\hline A3 & 2.50 & 3.00 & 5.00 & 3.00 \\
\hline A6 & 2.50 & 4.00 & 5.00 & 4.00 \\
\hline A2 & 3.50 & 4.00 & 2.00 & 5.00 \\
\hline
\end{tabular}

\subsubsection{Normalização da Matriz de Decisão (AN)}

Tabela 17 - Matriz de Decisão Normalizada.

\begin{tabular}{|l|l|l|l|l|}
\hline & \multicolumn{4}{|l|}{ Critérios } \\
\hline Alternativas & E1 & E2 & E3 & E4 \\
\hline A3 & 0.294118 & 0.272727 & 0.416667 & 0.25 \\
\hline A6 & 0.294118 & 0.363636 & 0.416667 & 0.333333 \\
\hline A2 & 0.411765 & 0.363636 & 0.166667 & 0.416667 \\
\hline
\end{tabular}

\subsubsection{Vetor de pesos dos critérios e Matriz Ponderada}

O novo vetor de pesos $(\mathrm{w})$ com os valores dos pesos arbitrados para os critérios para a segunda etapa é apresentado na tabela 18.

Tabela 18 - Vetor de Pesos(w).

\begin{tabular}{|l|l|l|l|l|}
\hline Vetor de Pesos & E1 & E2 & E3 & E4 \\
\hline W & 0.4546 & 0.091 & 0.182 & 0.273 \\
\hline
\end{tabular}


Tabela 19 - Matriz de Decisão Ponderada - segunda etapa.

\begin{tabular}{|c|c|c|c|c|}
\hline & Critérios & & & \\
\hline Alternativas & E1 & E2 & E3 & E4 \\
\hline $\mathrm{A} 3$ & 0.1336898 & 0.0247934 & 0.0757576 & 0.0681818 \\
\hline A6 & 0.1336898 & 0.0330579 & 0.0757576 & 0.0909091 \\
\hline A2 & 0.1871658 & 0.0330579 & 0.030303 & 0.1136364 \\
\hline
\end{tabular}

\subsubsection{Determinação da PIS(S+) e NIS(S-)}

A PIS(S+) e a NIS(S-) são determinadas, respectivamente, pelo maior e menor valor obtidos da matriz ponderada em cada critério. Os valores obtidos são apresentados na tabela 19.

Tabela 20 - Solução Ideal Positiva e Solução Ideal Negativa.

\begin{tabular}{|l|l|l|l|l|}
\cline { 2 - 5 } \multicolumn{1}{c|}{} & \multicolumn{4}{l|}{ Critérios } \\
\cline { 2 - 5 } \multicolumn{1}{c|}{} & E1 & E2 & E3 & E4 \\
\hline S+ & 0.1871658 & 0.0330579 & 0.0757576 & 0.1136364 \\
\hline S- & 0.1336898 & 0.0247934 & 0.030303 & 0.0681818 \\
\hline
\end{tabular}

\subsubsection{Cálculo das distâncias de cada alternativa para PIS(S+) e NIS(S-)}

O cálculo das distâncias euclidianas de cada alternativa Ai para $\mathrm{S}+$ e $\mathrm{S}$ - é dado pelas equações (3) e (4), respectivamente. A tabela 21 consolida os valores obtidos.

Tabela 21 - distâncias das alternativas para S+e S-.

\begin{tabular}{|l|l|l|l|}
\hline \multirow{2}{*}{ Distâncias } & \multicolumn{3}{|l|}{ Alternativas } \\
\cline { 2 - 4 } & A3 & A6 & A2 \\
\hline $\mathrm{d}+$ & 0.0706689 & 0.0581051 & 0.0454545 \\
\hline $\mathrm{d}-$ & 0.0454545 & 0.0514873 & 0.0706689 \\
\hline
\end{tabular}

\subsubsection{Cálculo da Similaridade}

A Similaridade (Ci) é dada pela equação (5), os resultados obtidos são apresentados na tabela 22 .

Tabela 22 - Similaridades das alternativas.

\begin{tabular}{|l|l|l|l|}
\hline & A3 & A6 & A2 \\
\hline Similaridade & 0.391433 & 0.469807 & 0.608567 \\
\hline
\end{tabular}

A partir das similaridades obtidas, as alternativas foram ordenadas em: A2, A6 e A3; sendo assim a alternativa A2, com maior valor de similaridade, foi selecionada para a composição da equipe.

\section{CONSIDERACOES FINAIS}

$\mathrm{Na}$ busca por uma solução para o problema de seleção múltipla foi conduzido uma análise experimental multicritério utilizando a técnica TOPSIS.

A primeira etapa do experimento proporcionou a eliminação de duas alternativas que não atendiam a restrições impostas pelo problema, quais sejam pretensão salarial (limitação de recursos) e distância da residência ao trabalho (minimizar riscos de faltas e atrasos). O resultado obtido com a ordenação pelo TOPSIS para esta etapa atendeu as restrições.

O modelo proposto na segunda etapa contemplou duas fases, tal abordagem permitiu 
a seleção das alternativas A1 e A2, que atendem satisfatoriamente ao requisito estabelecido para a seleção da equipe, complementaridade.

Caso fosse escolhido um modelo executado em apenas uma fase, as opções selecionadas seriam as alternativas A1 e A3, que aparentam grande similaridade entre si, representam alternativas substitutas.

Analisando as soluções propostas em ambos os modelos confirmam a hipótese que que a melhor opção de dupla não são as duas melhores colocadas na ordenação simples, mas sim aquelas que juntas representam o melhor conjunto, dados os critérios e condições estabelecidos no contexto do problema.

Como trabalhos futuros, sugere-se $r$ a obtenção de pesos para os critérios por meio do AHP.

\section{REFERÊNCIAS BIBLIOGRÁFICAS}

[1] Heizer, J.H.;Render, B. Administração de operações: bens e serviços. 2001: LTC.

[2] Alencar, L.H.;Almeida, A.T.d. A model for selecting project team members using multicriteria group decision making. Pesquisa Operacional, 2010. 30: p. 221-236.

[3] Al-Reshaid, K.;Kartam, N. Design-build pre-qualification and tendering approach for public projects. International Journal of Project Management, 2005. 23(4): p. 309-320.

[4] Alencar, L.H.;Almeida, A.T.d. Multicriteria decision group model for the selection of suppliers. Pesquisa Operacional, 2008. 28: p. 321-337.

[5] Al-Harbi, K.M.A.-S. Application of the AHP in project management. International Journal of Project Management, 2001. 19(1): p. 19-27.

[6] Kelemenis, A.; Ergazakis, K.;Askounis, D. Support managers' selection using an extension of fuzzy TOPSIS. Expert Systems with Applications, 2011. 38(3): p. 27742782.

[7] Lootsma, F.A. Introduction, in Multi-Criteria Decision Analysis via Ratio and Difference Judgement. 1999, Springer US. p. 1-14.

[8] Roy, B. Multicriteria Methodology for Decision Aiding. 1996: Springer.

[9] Sant'Anna, A.P.; Rabelo, L.M.;Nogueira, H.D. Comparação entre métodos multicritério em um modelo para avaliação da qualidade de ativos de renda variável. Revista de Finanças Aplicadas, 2012. 1: p. 1-21.

[10] Hwang, C.L.; Lai, Y.J.;Liu, T.Y. A new approach for multiple objective decision making. Computers and Operations Research, 1993. 20(8): p. 889-899.

[11] Hwang, C.L.;Yoon, K. Multiple attribute decision making: methods and applications : a state-of-the-art survey. 1981: Springer-Verlag.

[12] Liao, C.-N.;Kao, H.-P. An integrated fuzzy TOPSIS and MCGP approach to supplier selection in supply chain management. Expert Systems with Applications, 2011. 38(9): p. 10803-10811.

[13] Wang, W.-P. Toward developing agility evaluation of mass customization systems using 2-tuple linguistic computing. Expert Systems with Applications, 2009. 36(2, Part 2): p. 3439-3447. 
[14] Jozi, S.A., et al. An integrated Shannon's Entropy-TOPSIS methodology for environmental risk assessment of Helleh protected area in Iran. Environmental Monitoring and Assessment, 2012. 184(11): p. 6913-6922.

[15] Chen, W.;Wang, Z. Application of comprehensive evaluation methods in medical quality evaluation of clinical departments. Journal of Shanghai Jiaotong University (Medical Science), 2013. 33(7): p. 1018-1023.

[16] Boehm, B.W. Software risk management: Principles and practices. IEEE Software, 1991. 8(1): p. 32-41.

[17] Palharini, F.A. Recrutamento e Seleção de Pessoas: fundamentos e tendências. Cadernos do ICHF, 2008. 80(Série: Estudos e Pesquisas (UFF)). 